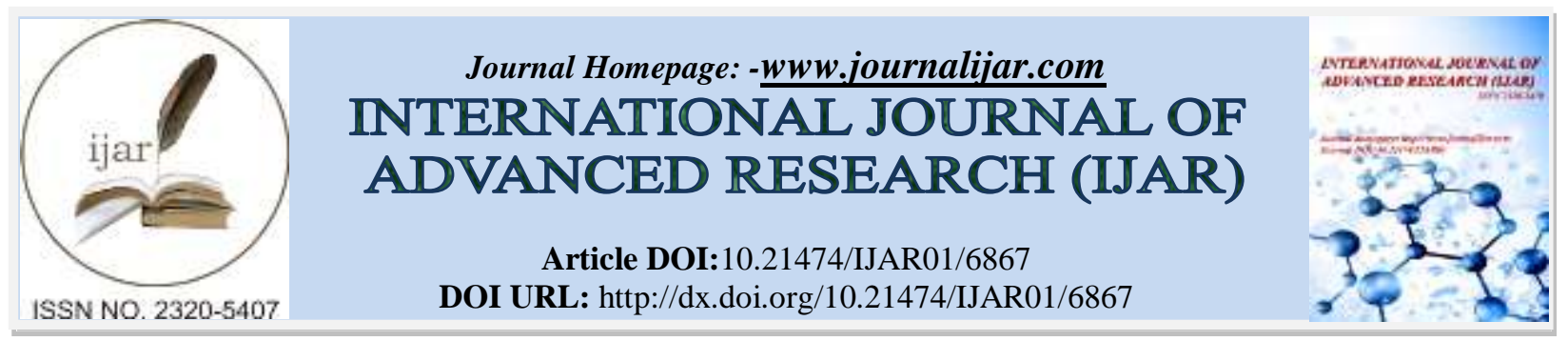

RESEARCH ARTICLE

\title{
EFFECT OF NESTING ON PHYSIOLOGICAL PARAMETERS AMONG PRETERM INFANTS ADMITTED IN NICUS.
}

\section{Sr. Mony k $\mathbf{k}^{1}$, Dr. Indra Selvam $\mathrm{V}^{2}$, Dr. Krishnakumar Diwakar ${ }^{3}$ and Dr. R. Vijaya Raghavan ${ }^{4}$.}

1. Professor ,Pediatric Nursing ,MOSC College of Nursing, MOSC Medical College hospital, kolenchery, Ernakulam dst, Kerala, India.

2. Principal and HOD Obstetrics and Gynaecology Nursing, Aswani College of Nursing ,Thrissur ,Kerala India

3. Dean and HOD Department of Neonatology MOSC Medical College, Kolenchery, Ernakulam, kerala, India

4. Director, Department of Research, Saveetha University, Chennai, Tamilnadu.

\section{Manuscript Info}

\section{Manuscript History}

Received: 06 February 2018

Final Accepted: 08 March 2018

Published: April 2018

Keywords:-

Physiological Parameters , Preterm Infants, Nesting

\section{Abstract}

Purpose: This study evaluated the effect of nesting on physiological parameters among preterm infants

Design and Methods: This crossover clinical trial was performed at a tertiary neonatal intensive care unit (NICU). 21 preterm infants who met the inclusion criteria were enrolled. They were randomly assigned to two groups of nest and routine procedure .Physiological parameters such as Oxygen saturation ,body temperature, heart rate and respiration were evaluated by using leads and probes connected to centralized monitor during each stages of sleep between inter epoch two feed were recorded and analyzed by using paired t-test

Results: There was improvement in physiological parameters among preterm infants with nesting compared to routine care, which was tested by paired $\mathrm{t}$-test. With regard to oxygen saturation, there was a significant increase in active sleep $(\mathrm{p}<0.05)$ and quiet sleep $(\mathrm{p}<0.005)$, heart rate was significantly decreased and maintained stable during quiet sleep $(\mathrm{p}<0.05)$ respiratory rate was decreased and maintained stable but statistically not significant( $p>0.05)$ in all stages of sleep , There was increase in mean body temperature value in all stages of sleep ,statistical significance exhibited only in indeterminate sleep $(\mathrm{p}<0.05)$.

Conclusion: Use of nesting aids in comfort and helps to maintain stable physiological parameters . Thus using nesting for preterm infant is recommended to facilitate stability of physiological parameters in NICU.

Copy Right, IJAR, 2018,. All rights reserved.

\section{Introduction:-}

Stable physiological parameters such as temperature, heart rate, respiratory rate and oxygen saturation are the indicators of good health especially among preterm infants.Sleep is important for the normal growth and development of an infant especially for the development of brain(AllenK ,2012, Calciolari G ,MontirossoR ,2011 Ardura etal 1995) Preterm infants have immature neurological functioning and need care in intensive unit for an extended period of time. Preterm infants born early with immature neurological functioning may live in the

Corresponding Author:-Mony k.

Address:-Professor, Pediatric Nursing, MOSC College of Nursing, MOSC Medical College hospital, 
neonatal intensive care unit (NICU) for an extended period of time. From birth on, these infants face a series of challenges in various areas of development. One of the major challenges in the first months of life is maintenance of stable physiological parameters.Stable physiological parameters reflects the maturation of the central nervous system (CNS) (Thoman 2002)Nesting may promote comfort and sleep there by able to maintain stable physiological parametrs

\section{Back ground:-}

Globally around 15 million preterm birth occurs (WHO 2014 )In India almost 3.6 million premature births (Indian Foundation for Premature Babies 2014 ) Statistics of KeralaShows-36.7\% ( Research Programme on Local Level Development report 2004) 1 million children die each year due to complications. Across 184 countries, the rate of preterm birth ranges from 5\% to $18 \%$ of babies born.(WHO2014) .( Nati ional Institute of Lung and Heart Disease, 2015) There are some studies shows that positioning supports promote sleep(Hideki kihara,Tomohiko Nkamura ,2013 ).There are some reports suggesting that use of the prone position with preterm infants can increase time in quiet sleep and decreases active sleep time and it can decrease time spent in awake (kulak Lo ,Ruggins NR,Stephenson TJ,1994).Insufficient sleep is associated with behavioral and cognitive deficits (Astill,Van der Heijden,Van Ijzendoom and Van Someren 2012 ).016 )Protecting comfort of an infant is a critical component of providing developmentally appropriate care for premature and full-term infants in the neonatal intensive care unit (NICU) because many of these infants are hospitalized during one of the most critical periods of brain development. (Kimberly A. A,2008) Nesting facilitates reduction in the discomfort (Remya ,2015)Nesting facilitates transformation of sleep pattern from erratic disturbed spells to deep peaceful nights and contented days thus conserving energy and minimizing weight loss (Prasanna, K., \& M, Radhika. (2015)

Camelia Mendes da silva,(2013) conducted a non controlled clinical trial among 12 newborns infants on physiological responses of preterm preterm newborn infants submitted to musical therapy(Rev.paul ,2013) Intants with gestational age $\leq 36 \mathrm{wks}$ and spontaneously breathing were included in the study The preterm infants were submitted to 15 minutes sessions of classical music therapy twice a day ( morning and afternoon )for three consecutive days .The variables heart rate and respiratory rate, oxygen saturation, diastolic and systolic arterial pressures and body temperature were analyzed before and immediately after each musical session .Result showed that there was a decrease in heart rate after the second session of music therapy (paired t-test $; \mathrm{p}=0.02$ ) and an increase at the end of the third session (paired t-test $; \mathrm{p}=0.005$ )Respiratory rate decreased during the fourth and fifth session (paired $\mathrm{t}$-test $; \mathrm{p}=0.01$ and 0.03 respectively)Regarding oxygen saturation ,there was an increase after the fifth session ( $\mathrm{p}=0.008$ ).comparison of physiological parameters among sessions, for the six studied sessions, showed only that the gain in oxygen saturation during the fifth session was significantly higher than during the sixth one (Turkey's test after variance analysis $: \mathrm{p}=0.04$ ) Conclusion: Music therapy may modify short term physiological responses of hospitalized preterm newborn infants.

Alpanamayi Bera (2014)conducted a study on effect of kangaroo Mother care on Vital physiological Parameters of low birth weight newborn .study conducted among 265 mother baby pairs .sample selected by purposive sampling .Initially KMC was started for 1hour duration (at a stretch )on first day and then increased by one hour each day for next 2 days . Axillary temperature, respiration rate, heart rate $(\mathrm{Hr} / \mathrm{min})$ and oxygen saturation $(\mathrm{SpO} 2)$ were assessed for 3 consecutive days, immediately before and after KMC. Result showed that mean temperature rose by about 4 degree $\mathrm{C}, \mathrm{RR}$ by 3 per minute , $\mathrm{HR}$ by $5 \mathrm{bpm}$ and $\mathrm{SpO} 2$ by 5\% following KMC session. Which was statistically significant $(\mathrm{p}<0.05)$

An experimental study was conducted by Mrs.Ramya paulose ,Molly Babu(2015)on effect of nesting on posture discomfort and physiological parameters of low birth weight infants in NICU of selected government hospital of Delhi .60 subjects, 30 in experimental group and 30 in control group were selected by by stratified sampling technique where infants were stratified in to three groups based on their birth weight (1.0 -1.5 kg , 1.5 to $2.0 \mathrm{~kg}) \mathrm{pre}$ test post test control group design was used in which nesting was provided in experimental group 9hours per day for 5 days .posture ,comfort and physiological parameters were assessed before and during application of nesting .Result showed that a significant improvement in posture $(\mathrm{t}=12.64)$ found among infants with nesting .A significant reduction in the discomfort was observed in experimental group as compared to control group $(\mathrm{t}=10.65)$. Infants with nesting experienced stable physiological parameters of heart rate $(\mathrm{F}=1.70, \mathrm{p}=0.16)$ respiratory $\operatorname{rate}(\mathrm{F}=1.26, \mathrm{p}=0.29)$ and temperature $(\mathrm{p}<0.050)$ 
Another study was conducted by Kihara H ,Nakamura T(2013), on the effect of nested and swaddled positioning support in the prone position on heart rate,sleep distribution and behaviour state in very low birth weight infants .the study concluded that a prone position with nested and swaddled positioning support might facilitate sleep and heart rate stability compared to prone positioning alone.

Another study conducted by Sandra Maria Scheler Cardoso (2015) ) on new born physiological responses to noise in the neonatal unit among 61 low weight newborns admitted in neonatal units and in incubator. That was a prospective ,observational quantitative exploratory ,descriptive study. Data collected by using assessment of Preterm Infant Behavior Scale and noise analyzer from September 2012 to April 2013 .Result showed that there was increase in heart rate $(\mathrm{p}=0000 \pm 10.36 \mathrm{sd}$, when sleeping and 8.7 in noise ,respectively)and decrease of oxygen saturation in newborns in incubators, in the presence of higher environmental noise levels $(\mathrm{p}=0.0000$ and $\mathrm{Sd}$ of $1.05 \mathrm{and}$ of 1.12 ,when sleeping and in noise respectively).conclusion : there was high sound pressure levels in the NICU .There was variation in physiological or functional changes due to the effect of noise. There are very limited number of studies conducted on effect of nesting on physiological parameters . So the investigator felt the need to conduct a study on this area of interest

\section{Methods:-}

\section{Participants and Setting:-}

This is a randomized controlled clinical trial to assess the effect of nesting on physiological parameters among preterm infants, conducted in cross over design to control confounding factors of sleep of preterm infants .The study was conducted from March 2017 to May 2017 in NICU of MOSC Medical College ,Kolenchery , the selection of setting was purposive in nature. The selection of study setting was carried on the basis of feasibility of conducting the study, acquaintance of the researcher with the area, availability of participants and the co operation from the management of the Hospital. The Neonatal intensive care unit of MOSC Medical College hospital cater services to all neonates born within the hospital and referred cases from different parts of Kerala. This is a third level NICU provide care to Preterm and term neonates with all types of critical illness. Sample size estimated as 21 based on sample size formula. Inclusion criteria were Preterm infants with weight between $1.2 \mathrm{~kg}$ to $2 \mathrm{~kg}$, Infants with post conception age between 30 and 36 weeks, infants admitted in NICU,preterm infants with spontaneous breathing with no need of assisting device ,Having no major congenital defects or abnormal neurological findings including intra ventricular hemorrhage, no consumption of illicit narcotics or additive substances by the mothers during pregnancy and infants not treated with sedatives $24 \mathrm{hr}$ prior to intervention. Subjects were selected by random convenient method (drawing of lots) in such a way that every day a subject was selected until it reaches the sample size from the hospitalized infants meeting the inclusion criteria and evaluated. After obtaining ethical clearance from IEC of Saveetha university and MOSC Medical College hospital permission from Medical Superintendent of the hospital and written informed consent from the parents of preterm infants, the study subjects who met all inclusion criteria were selected by simple random method (drawing of lots) in such a way that every day ,one subject was randomly selected. Then these Subjects were randomly allocated for nesting and routine procedure of wrapping(through tossing a coin). After obtaining an informed written consent from parents of infants Socio demographic data extracted from their medical record .Each infant was put on nesting or routine care of wrapping method as per random allocation and observed between inter epoch of two feed .Feeding Interval of ICU was 2hour. After one intervention next two hours infant was kept on wash out period for 2 hours to prevent carry over effect of first intervention and then same subject was kept on second intervention. These infants were allowed to sleep after feed, physiological parameters such as oxygen saturation, heart rate ,respiratory rate and temperature were observed and recorded during sleep waking period between inter epoch of two feed by using leads and probes connected to centralized monitor.

\section{Instrument:- \\ Tools and technique:-}

Tool 1:- Structured questionnaire to collect demographic variables

Tool 2:- Data sheet for Physiological parameters such as heart rate ,respiratory rate and oxygen saturation and temperature which were observed by leads and probe connected to centralized monitor.

Socio demographic variables: this collected by using Semi structured questionnaire .This consist of Age ,Sex, post conception age and birth weight. Content validity of the tool were obtained by giving to experts in the field of neonatology, sleep centres and Nursing. Semi structured questionnaire to assess socio demographic data were translated in to Malayalam and retranslated in to English .Reliability of the tool tested by test retest method. 


\section{Data analyses:-}

Data were analyzed by SPSS 20 version

\section{Results:-}

Out of 21 infants in the study 12 were male and 9 were female .Mean of gestational age of study subjects were $32.81 \pm 1.365$ weeks .The mean of post natal age of study subjects were $6.38 \pm 5.343$ days Mean birth weight of the subjects were $1.67 \pm 0.1849$.

Table 1:-Comparison of physiological parameters in stages of sleep in nesting Vs routine care among preterm infants

\begin{tabular}{|l|l|l|l|l|}
\hline Variables & Mean & \multicolumn{2}{l|}{ Standard deviation } \\
\hline & Nesting & Routine care & Nesting & Routine care \\
\hline Oxygensaturation & & & & \\
\hline Active sleep & 98.52 & 97.19 & 1.289 & 1.401 \\
\hline Quiet sleep & 98.52 & 97.67 & 1.250 & 1.197 \\
\hline Indeterminate sleep & 98.00 & 97.81 & 1.897 & 1.692 \\
\hline Heart rate & & & & 11.710 \\
\hline Active sleep & 140.67 & 146.71 & 12.496 & 16.539 \\
\hline Quiet sleep & 135.81 & 142.62 & 11.786 & 13.665 \\
\hline Indeterminate sleep & 139.29 & 142.14 & & 6.877 \\
\hline Respiratory rate & & & 5.900 & 5.563 \\
\hline Active sleep & 51.29 & 50.90 & 7.658 & 5.088 \\
\hline Quiet sleep & 47.62 & 49.05 & 5.932 & \\
\hline $\begin{array}{l}\text { Indeterminate } \\
\text { sleep }\end{array}$ & 49.65 & 55.65 & & 0.3746 \\
\hline BodyTemperature & & & & 0.3442 \\
\hline Active sleep & 36.72 & 36.767 & 0.276 & 0.1599 \\
\hline Quiet sleep & 36.8319 & 36.705 & 0.332 & 0.157 \\
\hline $\begin{array}{l}\text { Indeterminate } \\
\text { sleep }\end{array}$ & 36.84 & 36.743 & & \\
\hline
\end{tabular}

Table .1 depicts that infants experienced stable physiological parameters in both procedures even though there was improvement during the period of nesting as compared to routine care in terms level of oxygen saturation was improved, heart rate decreased and maintained normal ,respiratory rate maintained stable ,body temperature increased to normal level with nesting as compared to routine procedure in all stages of sleep. This differences may be due to the effect of nesting.

Table2:-Effect of nesting on physiological parameters Oxygen Saturation ,heart rate ,respiratory rate and body temperature

\begin{tabular}{|c|c|c|c|c|c|}
\hline Variables & \multirow{2}{*}{$\begin{array}{l}\text { Mean } \\
\text { difference }\end{array}$} & \multirow{2}{*}{$\begin{array}{l}\text { Standard } \\
\text { deviation }\end{array}$} & \multirow[t]{2}{*}{ t- value } & \multirow[t]{2}{*}{$\mathrm{df}$} & \multirow[t]{2}{*}{ Sig(2-tailed) } \\
\hline With NestingVs Routine care & & & & & \\
\hline 1.SaO2 & \multirow[t]{2}{*}{1.333} & \multirow[t]{2}{*}{1.592} & \multirow[t]{2}{*}{3.839} & \multirow[t]{2}{*}{20} & \multirow[t]{2}{*}{0.001} \\
\hline Active sleep & & & & & \\
\hline Quiet sleep & 0.857 & 1.276 & 3.078 & 20 & 0.0061 \\
\hline Indeterminate sleep & 0.190 & 2.136 & 0.409 & 20 & 0.687 \\
\hline 2.Heart rate & \multirow[t]{2}{*}{-6.048} & \multirow[t]{2}{*}{14.441} & \multirow[t]{2}{*}{-1.919} & \multirow[t]{2}{*}{20} & \multirow[t]{2}{*}{0.069} \\
\hline Active sleep & & & & & \\
\hline Quiet sleep & -6.810 & 13.963 & -2.235 & 20 & $0.037 *$ \\
\hline Indeterminate sleep & -2.857 & 15.167 & -0.863 & 20 & 0.398 \\
\hline 3.Respiratory rate & \multirow[t]{2}{*}{0.381} & \multirow[t]{2}{*}{6.160} & \multirow[t]{2}{*}{0.283} & \multirow[t]{2}{*}{20} & \multirow[t]{2}{*}{0.780} \\
\hline Active sleep & & & & & \\
\hline Quiet sleep & -1.429 & 6.705 & -0.976 & 20 & 0.341 \\
\hline Indeterminate sleep & -6.000 & 26.121 & -1.027 & 20 & 0.317 \\
\hline 4.Body temperature & -0.0429 & 0.3443 & -0.570 & 20 & 0.575 \\
\hline
\end{tabular}




\begin{tabular}{|l|l|l|l|l|l|}
\hline \multicolumn{1}{|l|}{} & & & & & \\
\cline { 1 - 5 } Active sleep & & & & & \\
\hline Quiet sleep & 0.12714 & 0.44246 & 1.317 & 20 & 0.203 \\
\hline Indeterminate sleep & .1000 & .1924 & 2.382 & 20 & 0.027 \\
\hline
\end{tabular}

The data presented in Table 2 shows that there was improvement in physiological parameters, which was tested by paired $\mathrm{t}$-test .With regard to oxygen saturation there was a significant increase in active sleep ( $\mathrm{p}<0.05)$ and quiet sleep $(\mathrm{p}<0.005)$,heart rate was significantly decreased and maintained stable during quiet sleep $(\mathrm{p}<0.05)$, respiratory rate was decreased and maintained stable but statistically not significant $(p>0.05)$ in all stages of sleep ,in terms of body temperature, there was increase in mean body temperature value in all stages of sleep ,statistical significance exhibited only in indeterminate sleep $(\mathrm{p}<0.05)$. This indicate that nesting may promote comfort of the infant there by maintained stable vital physiological parameters.

\section{Discussion:-}

In Neonatal Intensive Care Unit preterm infants get disturbed due to the effect of many factors like noise ,light , lack of confined environment. This may be compensated by caring infant with nesting. In the present study showed that there was an improvement and stability in Physiological parameters like oxygen saturation ,heart rate, respiratory rate and temperature in all stages of sleep among infants with nesting as compared to routine care .This shows nesting may be effective in maintaining stable physiological parameters, which in turn an indicator of good health. This was supported by findings of the study conducted by Mrs. Ramya paulose ,Molly Babu(2015)on effect of nesting on posture discomfort and physiological parameters of low birth weight infants. The study concluded that infants with nesting experienced stable physiological parameters of heart rate, respiratory rate and temperature. Another study was conducted by Kihara H ,Nakamura T(2013), on the effect of nested and swaddled positioning support in the prone position on heart rate ,sleep distribution and behaviour state in very low birth weight infants .The study concluded that , a prone position with nested and swaddled positioning support might facilitate sleep and heart rate stability compared to prone positioning alone .

\section{Limitations:-}

Observation was done for small time period due to time boundaries of the study .

Limited sample size

\section{Implications for Nursing practice:-}

Nurse should engage in preventive and promotive services for enhancing health. The study finding helps a neonatal nurse to recognize the importance of identifying physiological parameters which is an indicator of health of infants. Neonatal intensive care nurse should impart a concept regarding sleep pattern of infants to the new mother and her support person during admission to postnatal ward for better adaptation to the disease phase.

\section{Conclusion:-}

The findings of the research study showed that nesting is effective in maintaining stable physiological parameters among infants admitted in neonatal intensive care units. This study findings can be utilized for caring preterm infants in neonatal intensive care units and in situation where there is lack in parental care to prevent the crippling complications like abnormal psychomotor and neurological sequelae that manifested by abnormal variations in physiological parameters.

\section{Acknowledgement:-}

We warmly thank all of the study participants and their parents and members of NICU for their cooperation in connection with this study.

\section{Human Subjects Approval Statement:-}

Institutional Ethical committee of Saveetha University and MOSC Medical College approved this research(Approval No 022/08/2016/IEC/SU and MOSC/IEC/239/2017)

\section{Funding:-}

Self funded 


\section{Declaration of conflicting Interest:-}

The authors have no conflicts of interest

\section{Reference:-}

1. Alpanamayi Bera ,jagabandhu ghosh ,Arun kumarendu Singh ,Avjith Hazra ,Tapas Som ,Effect of kangaroo mother care on vital physiological parameters of Low Birth Weight Newborn ,Indian journal of Community Medicine. 2014;39(4):245-249 Doi :10.4103/0970-0218 .143030

2. Anders TF, Sadeh A, Appareddy V. Normal sleep in neonates and children. In: Ferber R, Kryger M, editors. Principles and Practice of Sleep Medicine in the Child. 3rd ed. Philadelphia, PA: WB Saunders; 1995

3. Astill,Van der Heijden KB,Van Ijzendoom and Van Someren .Sleep ,cognition and behavioral problems in school age children :Acentury of research meta-analyzed .Psychological Bulletin .2012;138:1109View in article Cross ref Scopus (129)

4. Hideki Kihara ,Tomohiko Nakamura .Nested and swaddled positioning support in the prone position facilitates sleep and heart rate stability in very low birth weight infants ,Research and reports in Neonatology 2014:3 1114 DOI ://dx.doi.org/19,2147/RRN.S41292

5. Ingersoll EW,Thoman EB .Sleep/wake states of preterm infants :stability ,developmental change ,diurnal variation, and relation with caregiving activity, journal of child development 1999:70 1-10. 13.IOSR Journal of Nursing and Health Science ,2015,4(1)

6. Ramya Poulose ,Molly Babu ,Sharda rastogi, Effect of nesting on posture Discomfort and Physiological parameters of LowBirth Weight Infants, 2015 ,IOSR Journal of Nursing and Health Science ,ISSN : 2320 $1959 ; 4(1) 46-50$

7. Sheldon SH. Sleep in infants and children. In: Lee-Chiong TL, Sateia MJ, Carskadon MA, editors. Sleep Medicine. Philadelphia, PA: Hanley and Belfus Inc; 2002

8. Sandra Maria Scheler Cardoso (2015) Brazillian journal of Otorohinolarygology v81( 6 ), https ://doi.org /10.1016 /j.bjori 2014.11.008). 